\title{
Heme oxygenase- 1 expression protects the heart from acute injury caused by inducible Cre recombinase
}

Travis D Hull ${ }^{1,2,7}$, Subhashini Bolisetty ${ }^{1,3,7}$, Angela C DeAlmeida ${ }^{1,4}$, Silvio H Litovsky ${ }^{5}$, Sumanth D Prabhu ${ }^{1,4,6}$, Anupam Agarwal ${ }^{1,3,6}$ and James F George , $^{1,2,3}$

The protective effect of heme oxygenase-1 (HO-1) expression in cardiovascular disease has been previously demonstrated using transgenic animal models in which HO-1 is constitutively overexpressed in the heart. However, the temporal requirements for protection by HO-1 induction relative to injury have not been investigated, but are essential to employ HO-1 as a therapeutic strategy in human cardiovascular disease states. Therefore, we generated mice with cardiacspecific, tamoxifen (TAM)-inducible overexpression of a human HO-1 (hHO-1) transgene (myosin heavy chain (MHC)-HO-1 mice) by breeding mice with cardiac-specific expression of a TAM-inducible Cre recombinase (MHC-Cre mice), with mice containing an hHO-1 transgene preceded by a floxed-stop signal. MHC-HO-1 mice overexpress HO-1 mRNA and the enzymatically active protein following TAM administration $(40 \mathrm{mg} / \mathrm{kg}$ body weight on 2 consecutive days). In MHC-Cre controls, TAM administration leads to severe, acute cardiac toxicity, cardiomyocyte necrosis, and $80 \%$ mortality by day 3 . This cardiac toxicity is accompanied by a significant increase in inflammatory cells in the heart that are predominantly neutrophils. In MHC-HO-1 mice, HO-1 overexpression ameliorates the depression of cardiac function and high mortality rate observed in MHC-Cre mice following TAM administration and attenuates cardiomyocyte necrosis and neutrophil infiltration. These results highlight that HO-1 induction is sufficient to prevent the depression of cardiac function observed in mice with TAM-inducible Cre recombinase expression by protecting the heart from necrosis and neutrophil infiltration. These findings are important because MHC-Cre mice are widely used in cardiovascular research despite the limitations imposed by Cre-induced cardiac toxicity, and also because inflammation is an important

pathological component of many human cardiovascular diseases.

Laboratory Investigation (2013) 93, 868-879; doi:10.1038/labinvest.2013.74; published online 3 June 2013

KEYWORDS: cardiac inflammation; heart failure; heme oxygenase-1 (HO-1); inducible Cre recombinase

The heme oxygenase $(\mathrm{HO})$ family of enzymes catalyze the initial and rate-limiting step of heme catabolism, resulting in the formation of equimolar amounts of carbon monoxide (CO), iron, and biliverdin, which is subsequently converted to bilirubin by biliverdin reductase. ${ }^{1,2}$ Two $\mathrm{HO}$ isoforms have been described. Heme oxygenase-1 (HO-1) is inducible while $\mathrm{HO}-2$ is expressed constitutively. ${ }^{3} \mathrm{HO}-1$ is of particular interest because it is a potential therapeutic target for a number of diseases. In conditions resulting in cellular stress or tissue injury, including cardiovascular diseases such as ischemia/reperfusion injury and cardiac allograft rejection,
HO-1 expression is robustly induced as a protective response. ${ }^{4,5} \mathrm{CO}$ and bilirubin, products of heme catabolism by HO-1, have antiapoptotic, anti-inflammatory, and cytoprotective properties, partially due to the scavenging of reactive oxygen species and downregulation of proinflammatory cytokine production. ${ }^{6-8}$ Iron produced by heme degradation is diverted from the pro-oxidant Fenton reaction because it is sequestered by cellular ferritin, which is co-induced with HO- $1 .{ }^{9}$

Cre/loxP technology was adapted from the bacteriophage P1 to manipulate the expression of specific genes. Expression

\footnotetext{
${ }^{1}$ Department of Medicine, University of Alabama at Birmingham, Birmingham, AL, USA; ${ }^{2}$ Department of Surgery, University of Alabama at Birmingham, Birmingham, AL, USA; ${ }^{3}$ Nephrology Research and Training Center, University of Alabama at Birmingham, Birmingham, AL, USA; ${ }^{4}$ Comprehensive Cardiovascular Center, University of Alabama at Birmingham, Birmingham, AL, USA; ${ }^{5}$ Department of Pathology, University of Alabama at Birmingham, Birmingham, AL, USA and ${ }^{6}$ Department of Veterans Affairs Medical Center, Birmingham, AL, USA

Correspondence: Dr JF George, PhD, Division of Cardiothoracic Surgery, University of Alabama at Birmingham, 701 South 19 th Street, 790 Lyons-Harrison Research Building, Birmingham, AL 35294, USA.

E-mail: jgeorge@uab.edu

${ }^{7}$ These authors contributed equally to this work.

Received 8 March 2013; accepted 29 April 2013
} 
of the Cre recombinase protein causes excision of DNA sequences that are flanked (floxed) by loxP sites. ${ }^{10} \mathrm{~A}$ tissuespecific promoter can drive Cre recombinase expression, such that the floxed sequence is only deleted in a specific cellular compartment. However, long-term expression of Cre recombinase in cells or in tissues can result in toxicity due to alteration of proliferation, apoptotic pathways, and energy/metabolism. ${ }^{11-13}$ For example, it has been demonstrated that constitutive expression of Cre recombinase driven by the cardiac-specific $\alpha$-myosin heavy chain (MHC) promoter results in cardiac toxicity. ${ }^{14}$ In addition, constitutive Cre expression precludes the knockdown of genes that are embryonic lethal and even in mice that live to adult age, makes it possible for compensatory changes to occur at the cellular level that confound the interpretation of gene deletion studies. To circumvent this problem and to achieve temporal control of Cre/loxP genetic recombination in the heart, Sohal et al ${ }^{15}$ developed a mouse model in which Cre recombinase is flanked on either side by a mutated estrogen receptor (mer) ligand-binding domain and restricted to cardiac-specific expression using the $\alpha$-MHC promoter (Myh6-MerCreMer, hereafter referred to as MHC-Cre). The Cre recombinase-mer fusion protein is insensitive to endogenous estrogen, but binds specifically to tamoxifen (TAM), a selective estrogen receptor modulator. Following TAM administration, Cre recombinase is released from cytosolic sequestration by Hsp90 and translocates to the nucleus to mediate recombination at loxP sites. ${ }^{16-18}$ Numerous groups have used MHC-Cre mice to achieve cardiac-restricted TAMinducible gene knockdown. However, recently, it was reported that induction of MHC-Cre with TAM causes severe systolic dysfunction and compensatory cardiac hypertrophy by decreasing cardiac mitochondrial ATP production and altering the expression of genes involved in calcium handling. ${ }^{19,20}$

MHC-Cre mice can be used to achieve cell-specific overexpression of a gene when they are bred with transgenic mice containing a floxed-stop signal followed by the gene of interest downstream of a highly active promoter. In the resulting double transgenic mice, the gene of interest is only expressed after administration of TAM. Here, we generated such a mouse model to study the effect of cardiac-specific HO- 1 overexpression in acute cardiac injury using the MHCCre mouse to achieve cardiac-specific, temporally regulated induction, and overexpression of a human HO-1 (hHO-1) transgene. We report that overexpression of cardiac-specific HO-1 inhibits acute systolic dysfunction and cardiac injury in MHC-Cre mice treated with TAM and reduces cardiac infiltration by neutrophils.

\section{MATERIALS AND METHODS}

\section{Animals}

Male and female mice between 10-25 weeks of age were used in all experiments. Three transgenic mouse lines were utilized: (1) chicken $\beta$-actin (CBA)-flox mice, containing a hybrid CBA promoter upstream of a floxed $\beta$-galactosidase gene $(\beta$-gal $)$ and stop-codon cassette that is followed by the hHO-1 gene (hmox-1); (2) MHC-Cre mice (Jackson Labs; Bar Harborm, ME, USA; number 005650) with cardiac-specific Cre recombinase that is TAM-inducible; ${ }^{15}$ and (3) MHC-HO-1 mice, which were generated by mating CBA-flox mice with MHC-Cre mice to achieve cardiac-specific TAMinducible hHO-1 overexpression. CBA-flox mice were generated using the pCMV flox vector (a generous gift from Drs MJ Moeller and L Holzman, University of Michigan, Ann Arbor, MI). This vector contains the hybrid promoter with an enhancer element and a chimeric intron to enhance the in vivo activity of the promoter. ${ }^{21}$ The hHO-1 gene (hmox-1) cDNA was inserted downstream of the promoter and $\beta$-gal-stop-codon cassette. The CBA-flox construct was microinjected into F2 hybrid eggs from C57BL/6 F1 mice at the UAB transgenic core facility, and mice were screened for the construct with $\beta$-gal-specific primers. DNA isolated from tail was used to confirm the presence of the Cre and/or CBA-flox sequence in the transgenic mice used in this study. Primer sequences were as follows $\left(5^{\prime}-3^{\prime}\right)$ : CBA $5^{\prime}$-CGTACCGGTCATCATCACCATC ACCATTGACTCG- $3^{\prime}$ and $5^{\prime}$-TCTCTGCCTGGGTGTGCACC TCCTTGG- $3^{\prime}$; Cre $5^{\prime}$-GCCAGGCGTTTTCTGAGCATAC- $3^{\prime}$ and $5^{\prime}$-CACCATTGCCCCTGTTTCACTATC- $3^{\prime}$. MHC-HO- 1 mice were inbred for at least five generations before they were used for experiments.

TAM (Sigma) was dissolved in peanut oil by brief sonication and administered by intraperitoneal injection. A dose of $40 \mathrm{mg} / \mathrm{kg}$ body weight on 2 consecutive days (total dose of $80 \mathrm{mg} / \mathrm{kg}$ body weight) was used in all experiments. Studies were initiated 1 day after the second TAM administration. Animal care and manipulations for experimentation were conducted in accordance with the Guide for the Care and Use of Laboratory Animals and with approval of the UAB Institutional Animal Care and Use Committee.

\section{Transthoracic Echocardiography}

Echocardiography was performed as described previously. ${ }^{5}$ Briefly, mice were anesthetized with $1.5 \%$ isoflurane in $95 \%$ $\mathrm{O}_{2}$ and placed in the supine position. Body temperature was maintained at $36.5-37.5^{\circ} \mathrm{C}$ on a heated platform, and electrocardiograms and temperature were continuously monitored. Cardiac function was assessed (M-mode, and 2D) with a VisualSonics VeVo 770 Imaging System (VisualSonics, Toronto, ON, Canada) equipped with a high-frequency $30 \mathrm{MHz}$ probe. Data analysis was performed using VisualSonics software (VisualSonics). All parameters were measured in the parasternal long-axis view over at least five consecutive cardiac cycles and averaged from at least three measurements. These parameters include left ventricular (LV) end-diastolic diameter and end-systolic diameter (EDD and ESD), anterior and posterior wall thickness in systole and diastole, LV ejection fraction (EF), 
and heart rate. End-diastolic volume (EDV) and end-systolic volume (ESV) was measured using the modified Simpson's method. LV systolic function was indexed by LVEF (EF = $(\mathrm{EDV}-\mathrm{ESV}) / \mathrm{EDV} \times 100)$ and $\mathrm{LV}$ fractional shortening $(\mathrm{FS}=(\mathrm{EDD}-\mathrm{ESD}) / \mathrm{EDD} \times 100)$.

\section{HO-1 Gene Expression}

Total RNA was isolated from ventricular myocardium using TRIzol reagent (Invitrogen), according to the manufacturer's protocol. The RNase-Free DNase Set (Qiagen) was used to digest contaminating genomic DNA in the RNA sample and RNA cleanup was performed using the RNeasy kit according to the manufacturer's protocol (Qiagen). One microgram of total RNA was converted to cDNA using the QuantiTect Reverse Transcription Kit (Qiagen), and quantitative realtime PCR was performed with SYBR Green Mastermix (Invitrogen) and $10 \mathrm{pmol}$ primers for $\mathrm{mHO}-1$, hHO-1, and mouse GAPDH. Primer sequences were as follows $\left(5^{\prime}-3^{\prime}\right)$ : mouse HO-1, 5'-GGTGATGGCTTCCTTGTACC- $3^{\prime}$ and $5^{\prime}$-AGTGAGGCCCATACCAGAAG-3'; hHO-1, 5' -CATG ACACCAAGGACCAGAG- $3^{\prime}$ and $5^{\prime}$-AGTGTAAGGACCCAT CGGAG-3'; mouse GAPDH, 5'-ATCATCCCTGCATCC ACT- $3^{\prime}$ and $5^{\prime}$-ATCCACGACGGACACATT- $3^{\prime}$. Relative expression of mHO-1 and hHO-1 was normalized to mouse GAPDH as an internal control and quantified using the $\Delta \Delta \mathrm{Ct}$ method. Samples were performed in triplicate.

\section{Western Immunoblotting}

Protein extraction and immunoblotting were performed as previously described. ${ }^{22}$ Briefly, ventricular cardiac tissue was homogenized in RIPA buffer, electrophoresed in a 10\% SDSpolyacrylamide gel, and transferred onto a Hybond C Extra membrane (Amersham Biosciences). Membranes were incubated with anti-HO-1 (1:5000 dilution; Stressgen) followed by a horseradish peroxidase (HRP)-conjugated goat anti-rabbit IgG secondary antibody (1:10000; Jackson ImmunoResearch Laboratories, West Grove, PA, USA), and HRP activity was detected by chemiluminesence. Membranes were then stripped and reprobed with anti-mouse GAPDH antibody to confirm equal loading and transfer.

\section{HO Enzyme Activity Measurement}

Activity of the $\mathrm{HO}$ enzyme in cardiac tissue was measured as a function of bilirubin generation, as previously described..$^{23}$ Briefly, tissue was homogenized in collection buffer $\left(200 \mathrm{mmol} / \mathrm{l} \mathrm{KH}_{2} \mathrm{PO}_{4}, 135 \mathrm{mmol} / \mathrm{l} \mathrm{KCl}, 0.1 \mathrm{mmol} / \mathrm{l} \mathrm{EDTA}\right.$, $\mathrm{pH}$ 7.4) by sonication and then centrifuged at $10000 \mathrm{~g}$ for $20 \mathrm{~min}$. The supernatant was collected for ultracentrifugation (100000 g, $4{ }^{\circ} \mathrm{C}, 60$ min.; Beckman L7-35 Ultracentrifuge, 70 Ti rotor, Indianapolis, IN, USA). The pellet was resuspended in activity buffer $\left(100 \mathrm{mmol} / \mathrm{l} \mathrm{KH_{2 }} \mathrm{PO}_{4}, 2 \mathrm{mmol} / \mathrm{l} \mathrm{MgCl}{ }_{2}, \mathrm{pH}\right.$ 7.4), and then used to determine the $\mathrm{HO}$ activity after addition of rat liver cytosol (source of biliverdin reductase; $2 \mathrm{mg}$ per assay), hemin $(20 \mu \mathrm{mol} / \mathrm{l})$, glucose-6-phosphate ( $2 \mathrm{mmol} / \mathrm{l})$, glucose-6-phosphate dehydrogenase $(0.2 \mathrm{U})$, and nicotinamide adenine dinucleotide phosphate $(0.8 \mathrm{mmol} / \mathrm{l})$. The reaction was incubated for $60 \mathrm{~min}$ at $37^{\circ} \mathrm{C}$ in the dark and the bilirubin formed was extracted with chloroform, measured at wavelengths of $464-530 \mathrm{~nm}$, and enzyme activity was expressed as picomoles of bilirubin formed per $60 \mathrm{~min} /$ $\mathrm{mg}$ of protein.

\section{Immunohistochemistry and Light Microscopy}

A mid-sagittal section of the heart was fixed in 10\% neutralbuffered formalin for $16 \mathrm{~h}$, stored in $70 \%$ ethanol, and embedded in paraffin. Sections $(5 \mu \mathrm{m})$ were deparaffinized in xylene solution and rehydrated. Antigen retrieval was performed in Trilogy solution $\left(35 \mathrm{~min}\right.$ at $95^{\circ} \mathrm{C}$ ). Endogenous peroxidase activity was blocked with $3 \%$ hydrogen peroxide. Sections were blocked in 5\% normal goat serum and incubated for $24 \mathrm{~h}$ with polyclonal rabbit anti-HO-1 antibody (1:1000, SPA-895, Stressgen) in PBST (PBS, 0.1\% Tween 20). Slides were incubated in peroxidase-conjugated goat antirabbit antibody for $1 \mathrm{~h}$ at room temperature. A VIP substrate kit (Vector Laboratories, Burlingame, CA, USA) was used according to the manufacturer's protocol to visualize HO-1 expression levels in the heart. Sections were dehydrated using xylenes and ethanol, and then mounted.

To assess the cardiac injury caused by TAM-mediate activation of Cre recombinase, $5 \mu \mathrm{m}$ sections of myocardium were stained with hematoxylin and eosin (H\&E). Cardiac pathology was assessed by a blinded reviewer from at least three mice from each group. $\beta$-galactosidase enzyme reactions using $\mathrm{X}$-gal reagent were performed on frozen sections of myocardium to detect expression of the CBA-flox construct. Tissue was embedded in optimal cutting temperature compound after fixation in $2 \%$ paraformaldehyde and overnight incubation in $20 \%$ sucrose solution. Sections $(5 \mu \mathrm{m})$ were incubated with reaction solution $(66 \mathrm{mM}$ $\mathrm{Na}_{2} \mathrm{HPO}_{4}, 33 \mathrm{mM} \mathrm{NaH} \mathrm{PO}_{4}, 2 \mathrm{mM} \mathrm{MgCl}_{2}, 5 \mathrm{mM} \mathrm{K}_{3} \mathrm{FeCN}_{6}$, $5 \mathrm{mM} \mathrm{K}_{4} \mathrm{FeCN}_{6}, 1 \mathrm{mg} / \mathrm{ml} \mathrm{X-gal)}$ at room temperature for $16-$ $24 \mathrm{~h}$, and then washed and mounted in glycerol. Images were captured using a Lecia DM IRB microscope (Leica Microsystems, Bannockburn, IL, USA) and Image-Pro Plus software (Media Cybernetics, Bethesda, MD, USA).

\section{Isolation of Cardiac Leukocytes}

Mice were anesthetized with isoflurane $\left(1.5 \%\right.$ in $\left.95 \% \mathrm{O}_{2}\right)$ and the heart was perfused with $10 \mathrm{ml}$ of ice-cold saline through the inferior vena cava. The heart was explanted and washed with PBS. Atria and the great vessels were removed and the myocardium was minced into small pieces and digested using 1.67 Wünsch U/ml Liberase DL (Roche Diagnostics, Indianapolis, IN, USA) in RPMI-1640 medium (HyClone, South Logan, UT, USA) for $1 \mathrm{~h}$ at $37^{\circ} \mathrm{C}$. PEB buffer (PBS, $2 \mathrm{mM}$ EDTA, 0.5\% BSA) was added to disrupt calcium-dependent cell-cell complexes and to inactivate the Liberase enzyme. Dissaggregation was completed by drawing the digested suspension through a $21 \mathrm{G}$ needle. The homogenate was passed through a $70-\mu \mathrm{m}$ nylon filter (Fisher) 
to remove undigested and fibrous material, and then centrifuged at $300 \mathrm{~g}$. The pellet was resuspended in buffered ammonium chloride to lyse red blood cells. After washing in PBS, cells were counted and used for experiments.

\section{Flow Cytometry}

Cells were incubated with anti-mouse CD16/32 for 10 min on ice to block nonspecific binding of phycoerythrin to $\mathrm{FC} \gamma 3$ receptors. Cells were initially stained with allophycocyaninconjugated CD45.2 (clone 104), anti-mouse major histocompatibility complex class II biotin (clone M5/114.15.2), phycoerythrin-conjugated anti-mouse Gr-1 (Ly6G, clone RB6-8C5), and fluorescein isothicyanate-conjugated antimouse CD11b (clone M1/70) or CD11c (clone HL3) for $30 \mathrm{~min}$ on ice. Cells were washed and stained with peridinin chlorophyll protein complex-conjugated streptavidin for an additional $30 \mathrm{~min}$ on ice. Isotype-matched, fluorescently conjugated antibodies of irrelevant specificity were used as controls. Antibodies for flow cytometry were purchased from eBioscience. Data acquisition was performed on a FACSCalibur flow cytometer (BD Biosciences) and results were analyzed using FlowJo Software (Tree Star, Ashland, OR, USA).

\section{Statistical Analysis}

Data are presented as mean \pm s.e.m. The Student's $t$-test was used for comparison between two groups. ANOVA and the Newman-Keuls post-test were used for analyses comparing more than two groups. Differences were considered statistically significant at $P<0.05$.

\section{RESULTS}

\section{Generation and Characterization of Mice with Cardiac- Specific TAM-Inducible HO-1 Overexpression}

Mice with cardiac-specific, TAM-inducible HO-1 expression (MHC-HO-1) were generated by breeding MHC-Cre mice with CBA-flox mice. X-gal staining was performed on histological sections to confirm expression of the $\beta$-gal gene in the hearts of CBA-flox mice using wild-type littermates as controls (Figure 1a). HO-1 induction in the heart of MHCHO-1 mice was assessed after administration of two doses of TAM $(40 \mathrm{mg} / \mathrm{kg}$ of body weight per dose) on consecutive days by intraperitoneal injection. Using human-HO-1-specific primers, induction of hHO-1 mRNA occurred 1 day after TAM, and overexpression peaked at day 3 (Figure $1 \mathrm{~b}$ ). TAM-mediated Cre activation also caused co-induction of endogenous mouse HO-1 (mHO-1), which was detected using mouse-specific primers (Supplementary Figure 1). Western blot using antibody with crossreactivity for both human and mouse HO-1 revealed similar induction kinetics of HO-1 overexpression (Figure 1c). These results indicate very low basal expression of $\mathrm{HO}-1$ in the heart and tight regulation of hHO-1 expression, as CBA-flox mice and untreated MHC-HO-1 mice do not express detectable levels of HO-1 protein. However, 5 days after TAM administration, cardiac expression of the HO-1 mRNA and protein decreases.
To confirm that the hHO-1 protein is enzymatically active, $\mathrm{HO}$ activity assays were performed using purified cardiac microsomal fractions from MHC-HO-1 mice and controls (Figure 1d). Peak HO activity in the heart coincides with maximal induction of the HO-1 mRNA and protein at day 3 . Immunohistochemistry of HO-1 expression in the heart of MHC-HO-1 mice after TAM administration showed robust HO-1 overexpression compared with TAM-treated CBA-flox mice or vehicle-treated controls (Figure 1e). Interestingly, the level of HO-1 overexpression in adjacent cardiomyocytes is heterogeneous (Figure 1e). No HO-1 induction was detectable in sections of the liver, spleen, kidney, and skeletal muscle (data not shown). Therefore, stop-codon excision within the CBA-flox construct and subsequent hHO-1 overexpression occurred only after TAM administration and only in the presence of the MHC-Cre fusion protein.

\section{Cre Activation but not TAM Alone Induces Endogenous mHO-1 and Transgenic hHO-1 in the Heart of MHC-HO-1 Mice}

To confirm that HO-1 overexpression in MHC-HO-1 mice is specifically the result of induction of $\mathrm{HO}-1$ due to Cremediated recombination, CBA-flox mice, which lack Cre recombinase, were treated with TAM or vehicle. $m H O-1$ gene expression in the heart was analyzed by real-time PCR to determine whether TAM alone induces endogenous mHO-1 expression (Figure 2a). There was no significant difference in mHO-1 induction after TAM compared with vehicle-treated controls $(P=0.273)$. Therefore, TAM alone does not induce endogenous $\mathrm{mHO}-1$.

Although TAM does not cause endogenous HO-1 expression, TAM-mediated Cre activation may induce mHO-1. Therefore, MHC-HO-1 and MHC-Cre (lacks the hHO-1 transgene) mice were treated with TAM or vehicle, and mHO- 1 and hHO-1 expression was analyzed in the heart by real-time PCR (Figure 2b). Cre activation by TAM caused significant induction of $\mathrm{mHO}-1$ in MHC-Cre and MHCHO- 1 mice $(P=0.008$ and $P=0.001$, respectively, relative to vehicle-treated controls). However, induction of hHO-1 only occurs in MHC-HO-1 mice, and this induction is over twofold greater than mHO-1. Thus, activation of Cre by TAM, but not TAM alone, causes transient induction of mHO-1 in mice containing the MHC-Cre construct, independent of the presence of a floxed sequence.

\section{HO-1 Transgenic Mice have Normal Baseline Cardiac Phenotypes}

Although HO-1 is an antioxidant and cytoprotective enzyme, overexpression of HO-1 in the heart of MHC-HO-1 mice could be toxic to cardiomyocytes, leading to their death, and thus, the observed decrease in hHO-1 expression at day 5 after TAM. Therefore, echocardiographic assessment of cardiac structure and mechanical function was performed in humanized HO-1 transgenic (HBAC) mice (Table 1). These mice constitutively overexpress hHO-1 in every organ system 

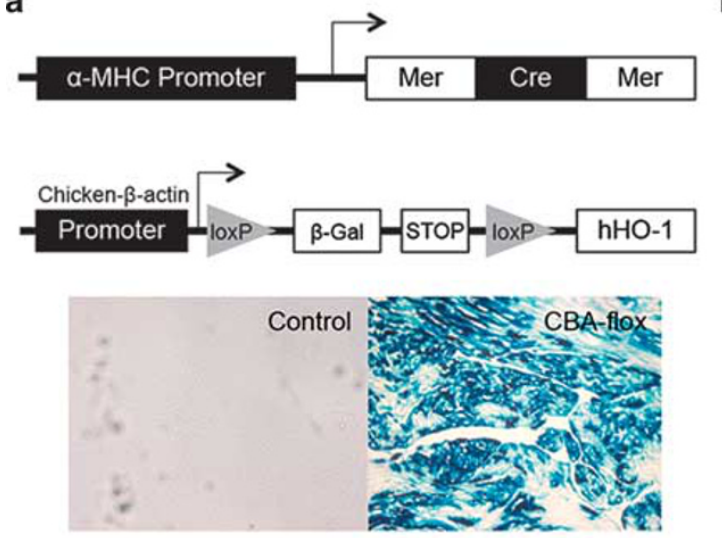

C

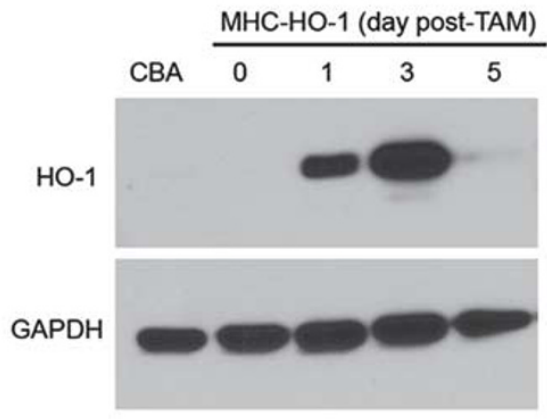

b

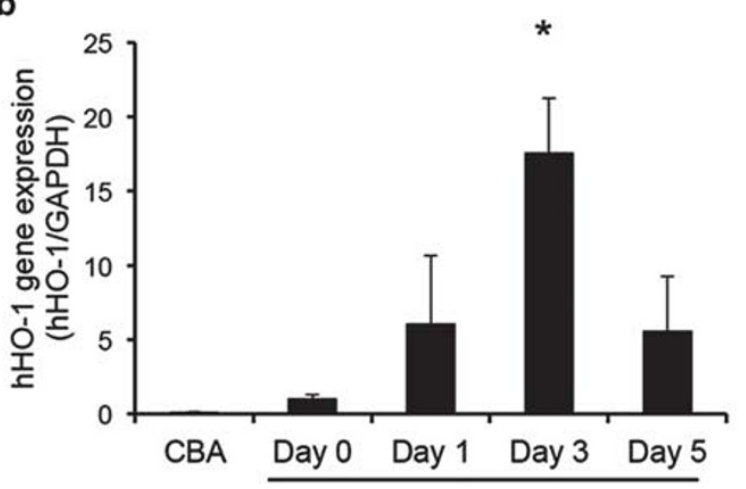

$\mathrm{MHC}-\mathrm{HO}-1$

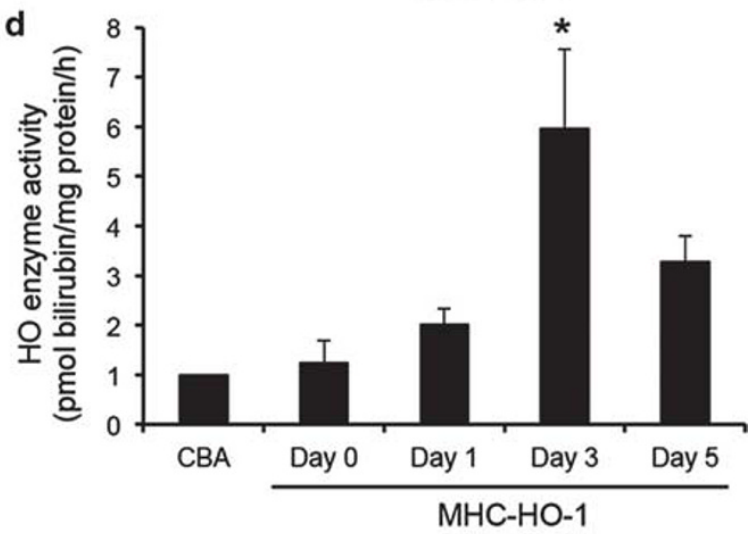

e

Vehicle
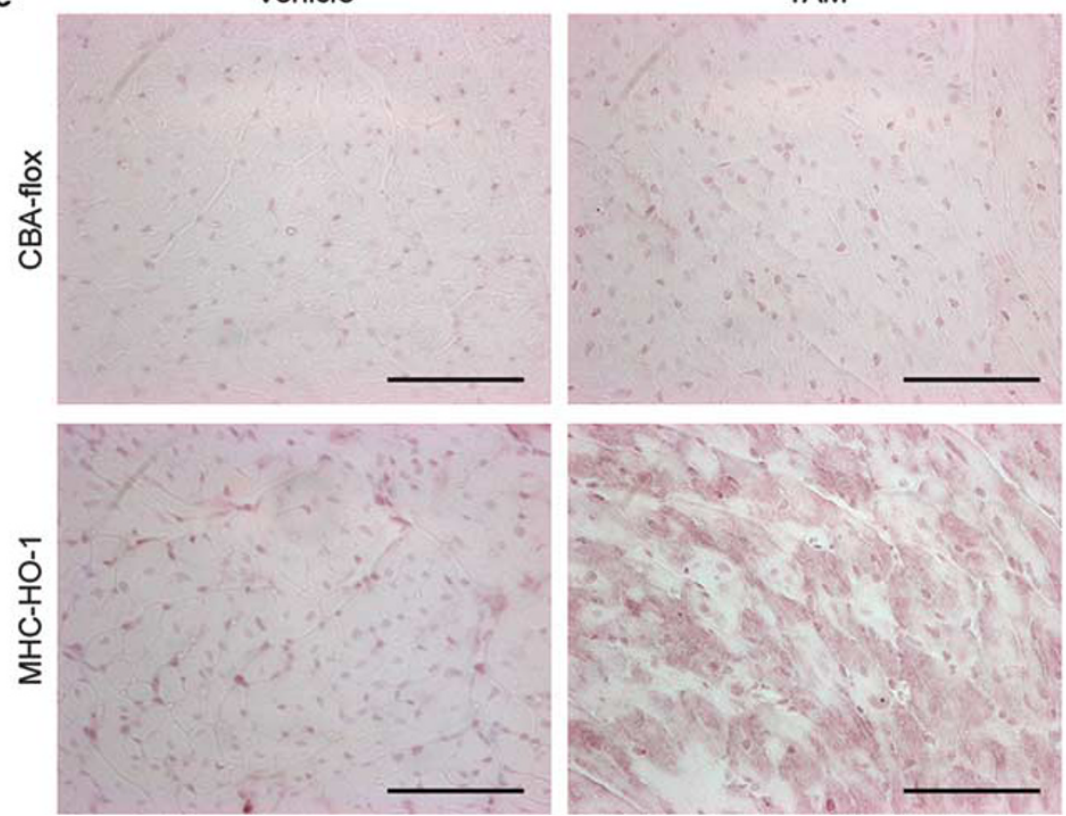

Figure 1 Generation and characterization of myosin heavy chain (MHC)-HO-1 mice. (a) Schematic of the transgenic constructs in MHC-Cre (top) and chicken- $\beta$-actin (CBA)-flox (bottom) mice that were used to generate MHC-HO-1 mice. $\beta$-galactosidase ( $\beta$-gal) staining on frozen sections of left ventricular (LV) myocardium from wild-type littermates (left panel) and CBA-flox mice (right panel) to verify cardiac expression of the $\beta$-gal transgene. (b) Real-time PCR analysis of cardiac human HO-1 (hHO-1) induction in MHC-HO-1 mice and CBA-flox controls following tamoxifen (TAM) administration. Results were normalized to GAPDH and expressed as average fold change ( \pm s.e.m.) compared with untreated (Day 0) MHC-HO-1 controls. (c) Heme oxygenase-1 (HO-1) protein expression by western blot analysis. GAPDH was used as a loading control. (d) HO enzyme activity in the microsomal fraction from cardiac tissue of MHC-HO-1 mice at the indicated times following TAM administration. Results are expressed as fold increase ( \pm s.e.m.) compared with control CBA-flox mice 3 days after TAM administration. (e) Representative immunohistochemical staining for HO- 1 expression on cardiac sections from CBA-flox and MHC-HO-1 mice administered vehicle or TAM. Scale bar, $100 \mu \mathrm{m} . n=3-6$ animals per time point. ${ }^{*} P<0.05$ versus control. 
a

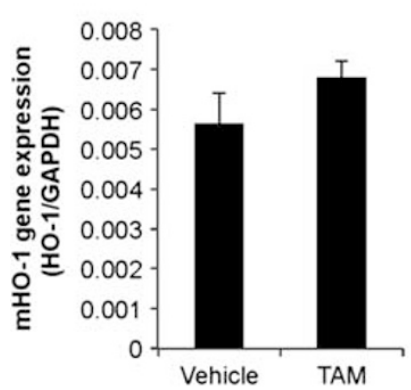

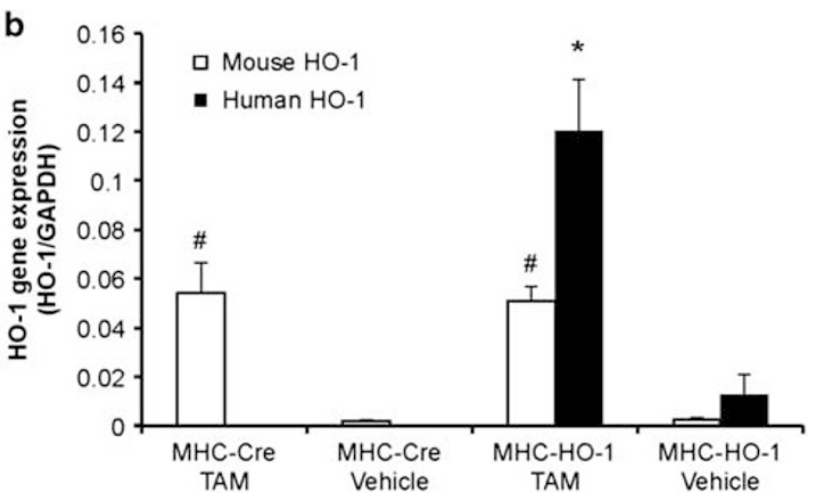

Figure 2 Tamoxifen (TAM)-mediated activation of myosin heavy chain (MHC)-Cre induces heme oxygenase-1 (HO-1). Real-time PCR analysis of (a) mHO-1 expression in the heart of chicken $\beta$-actin (CBA)-flox mice 2 days after administration of vehicle $(n=3)$ or TAM $(n=4)$; and $(\mathbf{b})$ mHO-1 and human HO-1 (hHO-1) expression in the heart of MHC-Cre and MHC-HO-1 mice treated with vehicle $(n=3-4)$ or TAM $(n=3-6)$. Analysis was performed in triplicate and results are normalized to GAPDH and expressed as an average \pm s.e.m. ${ }^{*} P<0.05,{ }^{\#} P<0.01$ versus vehicle.

Table 1 Baseline echocardiographic parameters of HO-1 transgenic mice and controls

\begin{tabular}{lccccc}
\hline & $\begin{array}{c}\text { Control }^{\mathrm{a}} \\
(n=9)\end{array}$ & $\begin{array}{c}\text { HBAC } \\
(n=4)\end{array}$ & $\begin{array}{c}\text { CBA }+ \text { TAM }^{\mathrm{b}} \\
(n=7)\end{array}$ & $\begin{array}{c}\text { MHC-Cre } \\
(n=7)\end{array}$ & $\begin{array}{c}\text { MHC-HO-1 } \\
(n=7)\end{array}$ \\
\hline BW & $22.9 \pm 1.42$ & $22.6 \pm 1.0$ & $23.7 \pm 1.50$ & $25.4 \pm 1.68$ & $22.9 \pm 1.57$ \\
ESV & $15.1 \pm 1.42$ & $18.1 \pm 1.19$ & $12.3 \pm 1.85$ & $15.1 \pm 1.15$ & $15.7 \pm 1.97$ \\
EDV & $46.2 \pm 7.21$ & $54.3 \pm 1.3$ & $44.9 \pm 2.27$ & $47.4 \pm 1.33$ & $46.0 \pm 3.76$ \\
ESD & $2.38 \pm 0.08$ & $2.30 \pm 0.06$ & $2.27 \pm 0.10$ & $2.24 \pm 0.03$ & $2.41 \pm 0.07$ \\
EDD & $3.6 \pm 0.09$ & $3.60 \pm 0.04$ & $3.54 \pm 0.08$ & $3.51 \pm 0.06$ & $3.64 \pm 0.08$ \\
AWTs & $0.83 \pm 0.02$ & $0.81 \pm 0.005$ & $0.89 \pm 0.03$ & $0.85 \pm 0.02$ & $0.87 \pm 0.01$ \\
AWTd & $0.68 \pm 0.01$ & $0.71 \pm 0.005$ & $0.71 \pm 0.01$ & $0.71 \pm 0.01$ & $0.72 \pm 0.01$ \\
PWTs & $1.0 \pm 0.01$ & $0.99 \pm 0.013$ & $1.11 \pm 0.05$ & $1.05 \pm 0.03$ & $1.02 \pm 0.02$ \\
PWTd & $0.67 \pm 0.01$ & $0.67 \pm 0.005$ & $0.74 \pm 0.03$ & $0.69 \pm 0.01$ & $0.71 \pm 0.01$ \\
FS & $34 \pm 0.96$ & $36.2 \pm 1.22$ & $36.2 \pm 1.57$ & $36.2 \pm 1.30$ & $34.0 \pm 0.91$
\end{tabular}

Abbreviations: BW, body weight (grams); ESV/EDV, end-systolic and end-diastolic volume $(\mu \mathrm{l}) ; \mathrm{ESD} / \mathrm{EDD}$, end-systolic and end-diastolic dimension $(\mathrm{mm})$; left ventricle AWT/PWT, anterior and posterior wall thickness $(\mathrm{mm})$ in systole (s) or diastole (d); FS, fractional shortening (EDD $-E S D) / E D D \times 100$.

Transgenic mice underwent echocardiography to characterize left ventricular structure and function.

Each parameter was measured in the parasternal long-axis view over $\geq 5$ cardiac cycles and averaged from at least three measurements.

Values are presented as average \pm s.e.m. None of the values were significantly different compared with the control.

antreated CBA.

${ }^{\mathrm{b}}$ Three days after TAM administration.

(20-fold in the heart), but are deficient for expression of mHO- $1 .{ }^{23}$ Compared with untreated CBA-flox controls, agematched HBAC mice exhibit no signs of cardiac dysfunction or structural remodeling. Therefore, it is unlikely that increased cardiac hHO-1 in MHC-HO-1 mice leads to cardiomyocyte death.

To confirm that TAM itself does not cause systolic dysfunction or structural remodeling, cardiac parameters were assessed by echocardiography at day 1,3 , and 5 in CBA-flox mice after treatment with TAM and compared with untreated controls (Table 1 and data not shown). TAM does not cause depression of cardiac function or structural changes in the heart. In addition, none of the parameters that were examined were significantly different in untreated MHC-Cre and MHC-HO-1 mice compared with controls. Therefore, neither TAM administration nor modulation of HO-1 expression alone affect cardiac function or cause cardiac remodeling.

\section{HO-1 Overexpression Prevents Lethal Cre-Induced Cardiac Toxicity}

It has recently been reported that TAM-mediated activation of Cre in MHC-Cre mice causes cardiac dysfunction that can be lethal. ${ }^{20}$ Therefore, survival of CBA-flox, MHC-Cre, and MHC-HO-1 mice was monitored following TAM administration (Figure 3a). Strikingly, TAM-mediated Cre-induction resulted in $80 \%$ mortality by day 3 in MHC-Cre mice. However, induction of the hHO-1 transgene in MHC-HO-1 mice provided a significant survival advantage, with a mortality rate of only $8 \%$ by day 3 and $16 \%$ by day 7 . TAM alone caused no mortality when administered to control CBA-flox mice.

Next, echocardiography was performed to monitor cardiac function after TAM administration (Figure $3 \mathrm{~b}$ and Table 2). In MHC-Cre-negative controls (CBA-flox mice), TAM administration did not cause a significant change in any of the parameters assessed. However, 1 day after TAM administration LV FS decreased significantly in MHC-Cre mice (23.75\%) but not in MHC-HO-1 mice (32.62\%) compared with CBA-flox controls (37.87\%). Mortality by day 3 precluded the further analysis of FS in MHC-Cre mice, whereas HO-1 overexpression in MHC-HO-1 mice prevented lethal cardiomyopathy and significant changes in FS up to day 5, relative to controls (Figure 3b). Echocardiographic analysis of the heart 1 day after TAM administration revealed that Cre causes LV chamber dilatation and thinning of the anterior and posterior (septal) walls. The extent of this remodeling is 

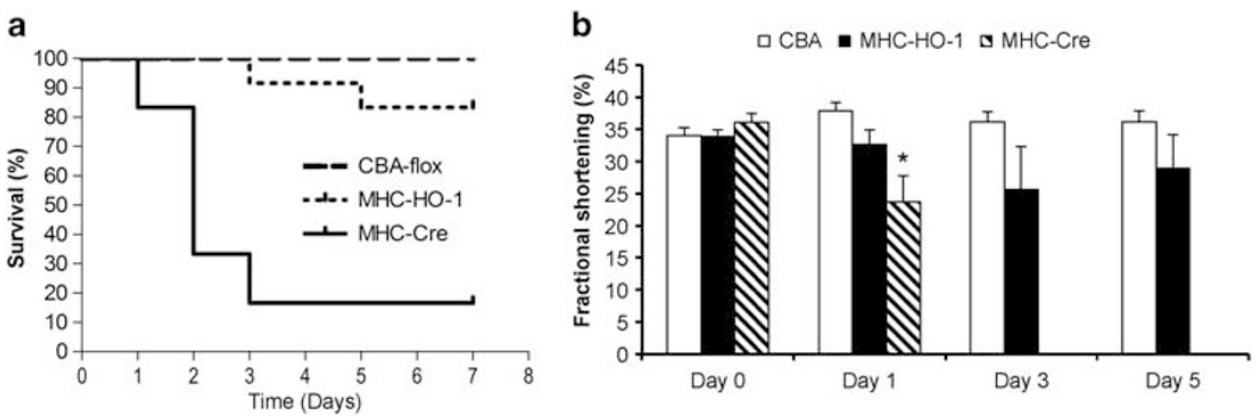

Figure 3 Heme oxygenase-1 (HO-1) expression prevents Cre-induced mortality and systolic dysfunction. Mice of the indicated strain were injected with tamoxifen (TAM). Mortality and cardiac function (indexed by fractional shortening (FS)) were monitored at the indicated times. (a) Kaplan-Meier plot showing survival after TAM administration ( $n=6-12$ per group). (b) Mice of the indicated group were monitored by echocardiography for changes in $\mathrm{FS}(\mathrm{FS}=(\mathrm{EDD}-\mathrm{ESD}) / \mathrm{EDD} \times 100)$ by comparing their FS before TAM treatment (Day 0$)$ with the indicated times after TAM treatment. All of the mice in the myosin heavy chain (MHC)-Cre group died before echocardiographic assessment on day 3. $n=5-7$ per group, ${ }^{*} P<0.05$ relative to control.

Table 2 Physical characteristics of cardiac structure after TAM administration

\begin{tabular}{lccc}
\hline & Control $(n=7)$ & MHC-Cre $(n=5)$ & MHC-HO- $(n=6)$ \\
\hline EDD & $3.51 \pm 0.08$ & $3.84 \pm 0.15$ & $3.70 \pm 0.14$ \\
ESD & $2.18 \pm 0.07$ & $2.85 \pm 0.28^{*}$ & $2.50 \pm 0.15$ \\
AWTd & $0.74 \pm 0.01$ & $0.67 \pm 0.03$ & $0.71 \pm 0.02$ \\
PWTd & $0.74 \pm 0.03$ & $0.71 \pm 0.05$ & $0.71 \pm 0.01$ \\
AWTs & $0.88 \pm 0.03$ & $0.79 \pm 0.05$ & $0.83 \pm 0.01$ \\
PWTs & $1.11 \pm 0.05$ & $0.97 \pm 0.08$ & $1.05 \pm 0.07$ \\
\hline
\end{tabular}

Abbreviations: EDD/ESD, end-diastolic and end-systolic diameter $(\mathrm{mm})$; left ventricle AWT/PWT, anterior and posterior wall thickness $(\mathrm{mm})$ in systole $(\mathrm{s})$ or diastole (d).

MHC-Cre and MHC-HO-1 mice were treated with TAM and parameters of cardiac structure were assessed after $24 \mathrm{~h}$ relative to controls (CBA-flox mice treated with TAM). Average \pm s.e.m. ${ }^{*} P<0.05$ relative to control.

decreased by HO-1 overexpression in MHC-HO-1 mice (Table 2). Thus, hHO-1 overexpression in MHC-HO-1 mice prevents Cre-induced acute systolic dysfunction and cardiac remodeling.

\section{HO-1 Prevents Severe Cardiac Inflammation and Myocyte Damage Caused by MHC-Cre}

To determine the cause of FS depression and mortality in MHC-Cre mice as well as the protective effect of HO-1, cardiac sections were harvested 2 days after treatment with TAM or vehicle and stained with H\&E (Figure 4). In mice lacking TAM-inducible Cre (CBA-flox), administration of TAM did not perturb normal myocardial cytoarchitecture or induce myocardial inflammation, compared with vehicletreated controls (Figure 4a-c). However, TAM administration to MHC-Cre mice resulted in severe, diffuse, interstitial inflammation of the myocardium and signs of myocyte damage, including cardiomyocyte cytoplasmic vacuolization, hypereosinophilia, and loss of myocyte striations. In addition, examination of intramyocardial arteries and the endocardium revealed plump endothelial cells, a common characteristic during active inflammation, and clusters of intravascular inflammatory cells (Figure $4 \mathrm{~d}-\mathrm{f}) .{ }^{24}$ Examination of the morphological characteristics of the cellular infiltrate revealed many cells with a multilobular nucleus, characteristic of polymorphonuclear leukocytes (neutrophils) (Figure 4f, inset). In MHC-HO-1 mice treated with TAM, there was no evidence of myocardial damage (Figure 4g-i) and only rare foci of inflammation (Figure 4i, inset). Toluidine blue staining indicated the presence of mast cells only in MHC-Cre mice treated with TAM, but not in vehicle-treated controls or CBA and MHC-HO-1 mice treated with TAM (data not shown). By H\&E staining, necrosis and inflammation were not observed in the kidney, skeletal muscle, and spleen (data not shown), indicating that the cause of mortality in MHC-Cre mice is due to cardiacspecific expression of the Cre recombinase.

\section{HO-1 Protects the Myocardium by Preventing Cre-Induced Infiltration of Neutrophils}

To quantitate the extent of inflammation in the heart caused by induction of Cre, cells isolated from MHC-Cre and MHCHO-1 myocardium were stained with CD45, a pan-leukocyte marker, and analyzed by flow cytometry (Figure 5a). There was no significant difference in the proportion of $\mathrm{CD} 45^{+}$ cells in the hearts of MHC-Cre versus MHC-HO-1 mice treated with vehicle. However, relative to vehicle, TAM-inducible Cre activation caused a threefold increase in CD45 ${ }^{+}$ cells in the myocardium of MHC-Cre mice ( $4.82 \pm 0.46$ versus $15.0 \pm 1.76 \%$, respectively; $P \leq 0.01, n=3-5)$. HO-1 induction abrogated Cre-induced cardiac inflammation, with no significant difference in the proportion of $\mathrm{CD} 45^{+}$cells in MHC-HO- 1 mice treated with TAM or vehicle $(3.71 \pm 0.95$ versus $3.54 \pm 0.19 \%$, respectively; $P=0.88, n=3$; Figure $5 \mathrm{~b}$ ). These data indicate that TAM-inducible Cre activation causes significant inflammation in the heart, which is prevented by induction of cardiac-specific hHO-1. Further characterization of $\mathrm{CD}_{4} 5^{+}$cells indicated that the majority of the infiltrating cells in TAM-treated MHC-Cre mice were $\mathrm{MHCII}^{-}$ 

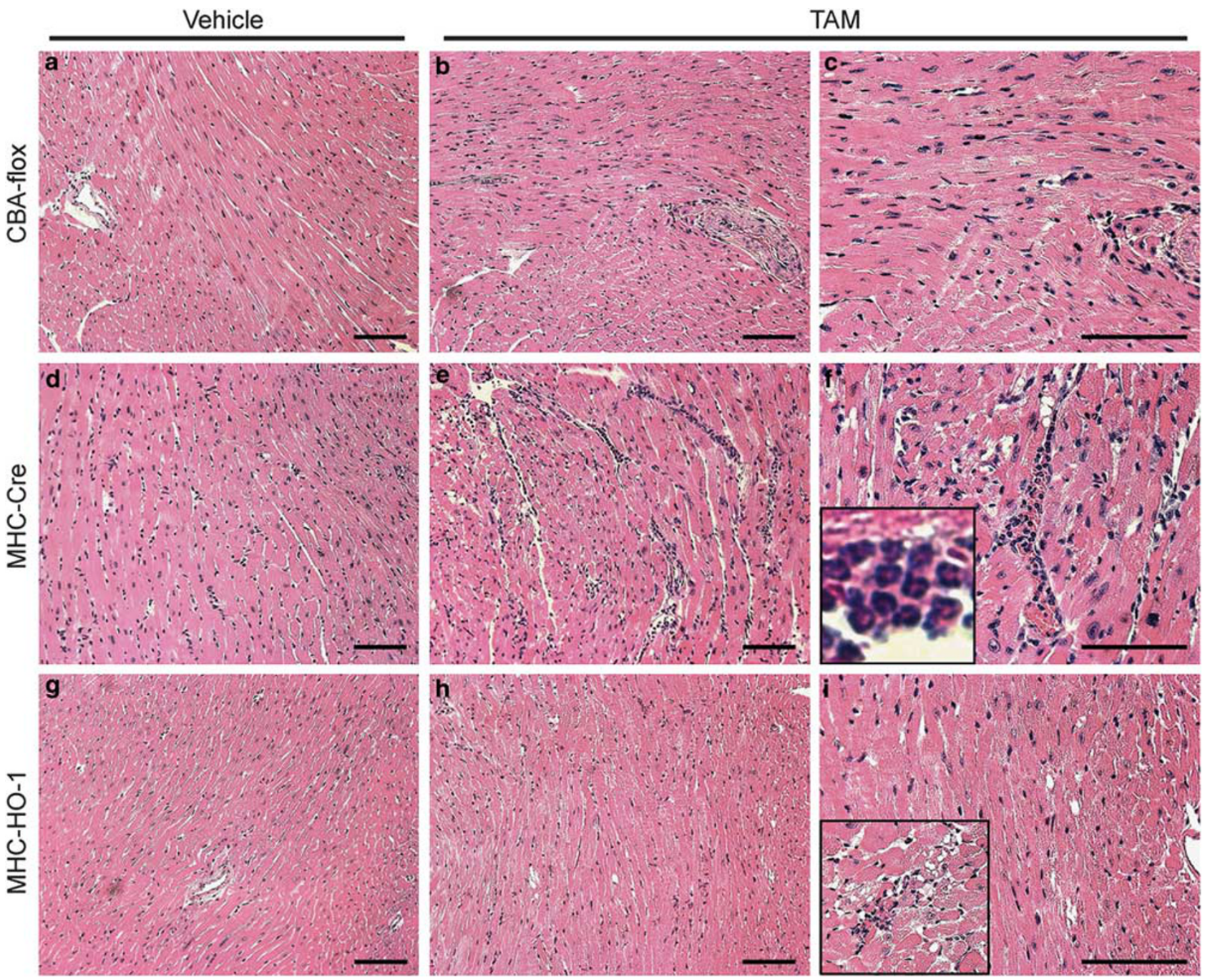

Figure 4 Heme oxygenase-1 (HO-1) overexpression protects the heart from Cre-mediated damage. Representative micrographs of mid-sagittal cardiac sections stained with hematoxylin and eosin (H\&E) from mice of the indicated group 2 days following tamoxifen (TAM) administration. (a) Vehicle or (b, c) TAM alone does not perturb normal cardiac cytoarchitecture in chicken $\beta$-actin (CBA)-flox mice. In myosin heavy chain (MHC)-Cre mice, (d) cardiac cytoarchitecture is normal after vehicle treatment, whereas Cre induction by TAM $(\mathbf{e}, \mathbf{f})$ causes transmural myocardial inflammation, hypereosinophilia, and cytoplasmic vacuolization of the myocardium. In MHC-HO-1 mice, neither vehicle (g) nor TAM (h, i) cause significant disturbances of normal cardiac cytoarchitecture, and only rare foci of inflammation are present (i; inset). Slides were assessed by a pathologist blinded to the different groups. Scale bar, $100 \mu \mathrm{m} . n=3-6$ per group.

Gr- $1^{+} \mathrm{CD} 11 \mathrm{~b}^{+}$neutrophils (Figure $5 \mathrm{c}$ ). This was an 18 -fold increase compared with vehicle-treated controls $(45.1 \pm 2.91$ versus $2.56 \pm 0.21 \%$, respectively; $P<0.001, n=3-5)$ and approximately a 7-fold increase compared with MHC-HO-1 mice treated with TAM $(45.1 \pm 2.91$ versus $6.33 \pm 0.21 \%$, respectively; $P<0.001, n=3-5$; Figure $5 \mathrm{~d}$ and e). Thus, HO-1 expression prevents neutrophil infiltration in response to TAM-mediated Cre activation.

Relative to MHC-Cre mice treated with vehicle, TAM administration caused a significant decrease in the proportion $(20.0 \pm 3.3$ versus $58.0 \pm 7.7 \%$; $P \leq 0.05)$ but not absolute number $(4125.2 \pm 1003$ versus $3313.3 \pm 770.4, P=0.54)$ of macrophages in the heart. There was no significant difference in the absolute number of cardiac macrophages or dendritic cells between MHC-Cre and MHC-HO-1 mice treated with vehicle $(3313.3 \pm 770.4$ versus $2041.3 \pm 98.3, P=0.18)$ or TAM (4125.2 \pm 1003 versus $1345.3 \pm 240.6, P=0.08$; Figure $5 f$ and $g$ and data not shown). Together, these results indicate that neutrophils, not monocytes/macrophages or dendritic cells, have a significant role in acute Cre-mediated cardiac toxicity.

\section{DISCUSSION}

We have demonstrated that cardiac-specific overexpression of HO- 1 is protective to the acute cardiac toxicity caused by TAM-inducible Cre recombinase. In accordance with previous studies, we show that TAM administration in MHC-Cre mice causes systolic dysfunction and dilated 
a

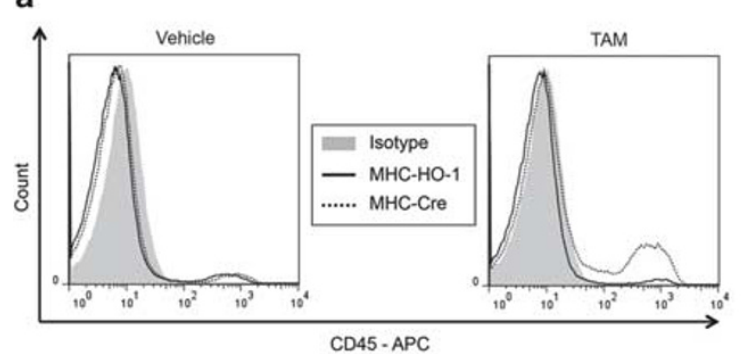

C b

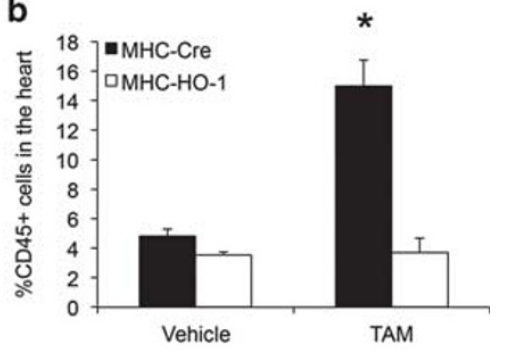

d
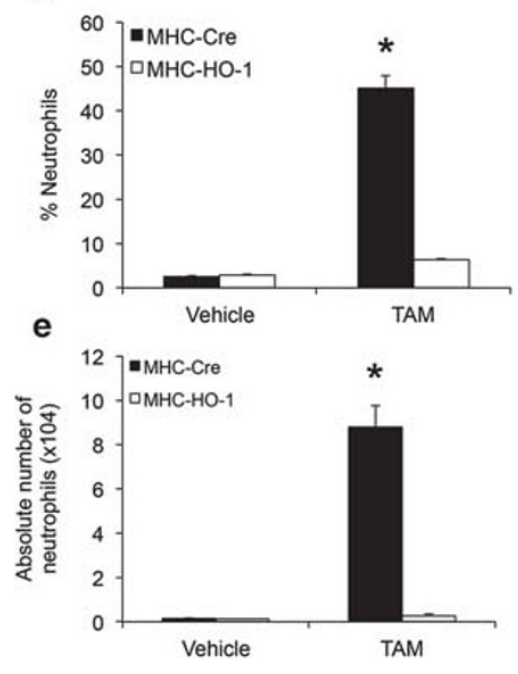

f

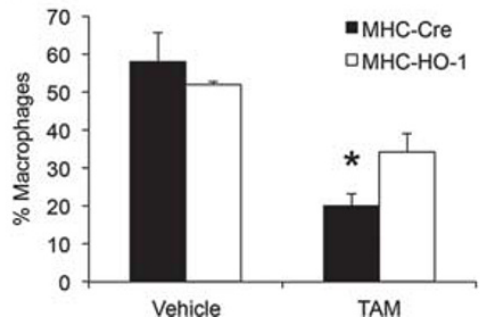

g

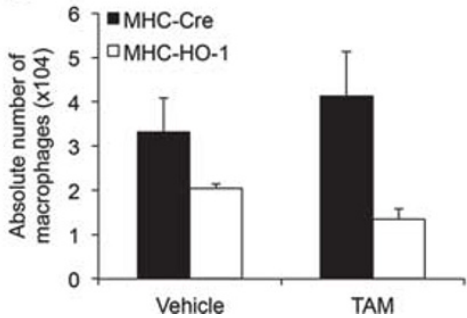

Figure 5 Heme oxygenase-1 (HO-1) overexpression prevents neutrophil infiltration after cardiac damage. Two days after treatment with tamoxifen (TAM) or vehicle, perfused hearts were explanted and homogenized for FACS analysis. Events $\left(6 \times 10^{5}\right)$ were collected per analysis. (a) Representative flow cytometry histograms of inflammatory cells in the heart identified as CD45 ${ }^{+}$and (b) quantified in myosin heavy chain (MHC)-Cre and MHC-HO-1 mice treated with TAM or vehicle. ${ }^{*} P \leq 0.01$ versus TAM-treated MHC-HO-1 and vehicle-treated control. (c) To characterize the cardiac infiltrate after TAM-mediated Cre induction, events in the forward and side scatter gate were analyzed for expression of CD45 and major hostocompatibility complex II (MHCII). Neutrophils were defined as CD45 ${ }^{+} \mathrm{MHCll}^{-} \mathrm{CD} 11 \mathrm{~b}{ }^{+} \mathrm{Gr}-1^{+}$. Neutrophils and macrophages $\left(\mathrm{CD} 45^{+} \mathrm{MHCll}^{+} \mathrm{CD} 11 \mathrm{~b}^{+}\right)$in the heart were also quantitated and results are presented (d, e) as a percent of the total number of $\mathrm{CD}_{4} 5^{+}$cells in the heart or in $(\mathbf{f}, \mathbf{g})$ as an absolute number per onethird of a total cardiac homogenate. $n=3-5$ animals per group in two separate experiments. Results are expressed as average \pm s.e.m. ${ }^{*} P \leq 0.001$ versus TAM-treated MHC-HO-1 and vehicle-treated control.

cardiomyopathy with $>80 \%$ mortality by day 3. ${ }^{19,20}$ TAMmediated Cre activation resulted in severe cardiac inflammation with prominent neutrophil infiltration and myocyte necrosis. In MHC-HO-1 mice, which overexpress cardiac-specific HO-1 after TAM administration, depression of cardiac function and subsequent mortality were attenuated, and cardiac inflammation and necrosis were prevented. In CBA-flox mice, TAM alone did not induce endogenous $\mathrm{mHO}-1$ expression. However, TAM-mediated activation of cardiac-specific Cre recombinase caused induction of endogenous mHO-1, independent of the presence of a floxed sequence. We have found that overexpression of $\mathrm{HO}-1$ prevents systolic dysfunction and cardiac injury in MHC-Cre mice by protecting the myocardium from Cre-induced toxicity. Our findings have several important implications because MHC-Cre mice are commonly utilized in cardiovascular research and because HO-1 is a potential therapeutic target in cardiovascular diseases that involve an inflammatory component, such as myocardial infarction. 
In the first report of MHC-Cre-mediated cardiac toxicity, Koitabashi et $a l^{20}$ demonstrated that Cre activation by TAM causes decreased expression of the genes responsible for mitochondrial energetics and biogenesis (ie, PPAR $\gamma, \operatorname{PGC}-1 \alpha$, and the TFAM genes). Hall et al ${ }^{19}$ then demonstrated decreased expression of PGC- $1 \alpha$ and PDK- 4 after TAM administration in MHC-Cre mice, which results in decreased production of mitochondrial ATP. Interestingly, in both of these studies, the nadir of systolic dysfunction occurred concomitantly with disturbances in the expression of these genes. Thus, although no definitive mechanism of Creinduced cardiac toxicity is known, it appears likely that Cre activation causes a shift in the metabolic activity of the cardiomyocyte from oxidative phosphorylation toward anaerobic respiration (ie, the glycolytic pathway), which has been shown to also occur in other forms of heart failure. ${ }^{25-27}$ Mitochondria comprise up to $35 \%$ of the cardiomyocyte cell volume because of the relatively high metabolic activity of the myocardium. Therefore, the heart is particularly susceptible to the insult caused by Cre activation. However, HO-1 and its byproduct, $\mathrm{CO}$ have been previously shown to prevent mitochondrial dysfunction and DNA depletion, and to upregulate mitochondrial biogenesis and ATP production in the heart. ${ }^{28-30}$ Thus, it is likely that HO-1 overexpression in MHC-HO-1 mice protects the heart from Cre toxicity directly by preventing mitochondrial dysfunction.

In accordance with our findings, it has been previously reported that cardiac inflammation occurs following TAM administration in MHC-Cre mice. The authors in these reports did not specifically characterize the cellular infiltrate, but speculated that it was due to an increase in cells from the mononuclear phagocyte lineage. ${ }^{20}$ As $\mathrm{HO}-1$ has been shown to protect the heart from injury due to its antiinflammatory properties, it is likely that $\mathrm{HO}-1$ provides a protective effect in Cre-induced cardiotoxicity by preventing inflammation. ${ }^{31,32}$ Here, we have demonstrated that TAMmediated Cre activation causes severe cardiac inflammation that directly precedes mortality and that the infiltrating cells are predominantly neutrophils. We have also shown that HO-1 overexpression prevents cardiac neutrophilia, suggesting that the anti-inflammatory property of the enzyme is at least partially responsible for its protective effect. The cardiac mononuclear phagocyte population is relatively unchanged by TAM-mediated Cre activation, as there are no significant differences in the number of macrophages or dendritic cells in the heart of MHC-Cre mice, following treatment with TAM or vehicle.

In our model, hHO-1 expression significantly decreases 5 days after TAM administration. Unlike the chemical inducers that are frequently used in animal models to achieve transient upregulation of endogenous HO-1 expression or activity, Cre/loxP genetic recombination is a stable event, and the hHO-1 transgene is downstream of the highly active hybrid chicken- $\beta$-actin promoter. Therefore, once maximal hHO- 1 induction is achieved, consistent levels of overexpression are expected over time. Although determining the cause of decreased hHO-1 expression is beyond the scope and intent of this report, several interesting possibilities exist. First, the cardiomyocytes that undergo Cre/loxP recombination and subsequently overexpress hHO-1 may undergo programmed cell death and/or necrosis, or be temporarily inactivated metabolically due to toxicity mediated by Cre. The former explanation is unlikely as we have shown that hHO-1 overexpression prevents cardiomyocyte necrosis 2 days after TAM administration. However, it is possible that sustained nuclear translocation of preformed MHC-Cre protein bound to TAM temporarily blocks hHO-1 expression by decreasing the generation of ATP needed for transcription of the transgene. Hougen et $a l^{33}$ recently demonstrated that TAM administration to MHC-Cre mice causes significant dysregulation of normal gene expression in the cardiomyocyte using microarray analysis. Consequently, it is also plausible that Cre-mediated alteration of gene expression causes decreased transcription of hHO-1. The MHC-Cre construct is expressed constitutively behind the highly active $\alpha$-MHC promoter, but Cre/loxP recombination is prevented because the construct is sequestered in the cytosol by Hsp90. ${ }^{16}$ Therefore, upon TAM administration, preformed Cre recombinase is immediately available. We have shown that TAM-mediated activation of Cre, and not TAM alone, induces the expression of endogenous HO-1, suggesting that it also causes a stress response in the cardiomyocyte. Therefore, it is possible that the massive increase in free Cre protein in the cell after TAM administration causes saturation of the ubiquitinproteasome system, endoplasmic reticulum stress, and/or alteration of normal nuclear import and export by saturating nuclear pore complexes, all of which could alter normal cellular homeostasis and thus result in decreased expression of the hHO-1 transgene after the initial induction.

Regardless of the cause of decreased hHO- 1 expression at day 5, overexpression of the hHO- 1 transgene at day 3 is sufficient to prevent MHC-Cre-mediated depression of cardiac function and resultant mortality by preventing cardiomyocyte necrosis and infiltration by neutrophils. Histological analysis demonstrated diffuse loss of the contractile apparatus and cardiomyocyte necrosis in MHC-Cre mice treated with TAM. These findings were absent in MHC-HO-1 mice, except for rare areas of focal inflammation. It is likely that foci of inflammation in MHC-HO-1 mice correspond to areas of the myocardium with low expression of the CBA-flox construct (Figure 1a), or low TAM delivery (and thus, Cre activation) to individual myocytes because of differences in the local microvasculature. Regardless of the cause of focal inflammation, our data indicate that there is no statistically significant difference in inflammatory cell number or cardiac function in MHC-HO-1 mice treated with TAM compared with controls. Collectively, these data suggest that HO-1 overexpression protects the myocardium from Cre-induced toxicity. 
Severe interstitial inflammation is likely a consequence of cardiomyocyte necrosis caused by cellular energy failure secondary to Cre activation. Intracellular components that are exposed to the extracellular environment due to necrotic cell death can be recognized by pattern recognition receptors on neutrophils as damage-associated molecular patterns (DAMPs). ${ }^{34-36}$ It has been shown previously that DAMP recognition by neutrophil pattern recognition receptors initiates a cascade of neutrophil activation, cytokine secretion, and recruitment of inflammatory cells including more neutrophils to the site of injury. ${ }^{37-39}$ Activated neutrophils secrete factors such as myeloperoxidase during their respiratory burst, which kill pathogens during infection, but also damage the surrounding host tissue. ${ }^{40,41}$ Therefore, it is likely that the cytoprotective properties of HO-1 prevent mitochondrial ATP depletion and subsequent cellular necrosis, which decreases the cardiac inflammatory response. Moreover, the anti-inflammatory properties of HO-1 directly provide an additional level of protection to the heart after TAM-mediated Cre activation.

In our model, early hHO-1 overexpression (days 1-3) was sufficient to prevent Cre-induced cardiac toxicity. Investigators employing MHC-Cre mice in cardiovascular research could utilize this finding because transient $\mathrm{HO}-1$ induction can easily be achieved using inducers such as hemin or cobalt protoporphyrin-IX. ${ }^{42,43}$ These inducers could be employed to prevent the acute Cre-mediated toxicity that occurs in MHC-Cre mice, which precludes investigation of the events that occur immediately as a result of the intended genetic manipulation. As compensatory changes may occur as a result of gene deletion during the period of cardiac recovery from TAMmediated Cre activation but before the initiation of studies, this is an important consideration. Furthermore, as we have shown that HO-1 induction prevents the dilated cardiomyopathy, depression of cardiac function, and cardiomyocyte necrosis that occurs in MHC-Cre mice, transient HO-1 induction could also prevent the confounding effect of compensatory cardiac hypertrophy demonstrated by Hall et $a l^{19}$ by limiting the initial cardiac damage caused by MHC-Cre.

It has been shown that HO-1 overexpression is protective in models of chronic heart failure induced by coronary ligation. In these models, cardiac-specific HO-1 overexpression improves survival and mechanical function by decreasing cardiomyocyte apoptosis and inflammation. ${ }^{5,44}$ However, the effect of early transient induction of HO- 1 at the time of injury has not been studied. This is especially relevant because others have shown that the early cellular infiltrate after infarction is predominantly composed of neutrophils, and we have shown that $\mathrm{HO}-1$ protects the heart from acute damage in MHC-Cre mice by preventing neutrophil inflammation. ${ }^{45,46}$ Therefore, our findings have important implications regarding the use of MHC-Cre mice in future studies as well as in the development of novel therapeutic strategies for human cardiovascular disease.

Supplementary Information accompanies the paper on the Laboratory Investigation website (http://www.laboratoryinvestigation.org)

\section{ACKNOWLEDGEMENTS}

This work was supported by NIH Grants R01 DK 083390 (to JFG and AA), the core resource of the UAB-UCSD O'Brien Center (P30 DK079337; to AA), and T32 HL007918 Training Program in Cardiovascular Pathophysiology (to TDH). AHA Grants 0655318B (to JFG) and 11POST7600074 (to SB).

\section{DISCLOSURE/CONFLICT OF INTEREST}

The authors declare no conflict of interest.

1. Tenhunen R, Marver HS, Schmid R. Microsomal heme oxygenase. Characterization of the enzyme. J Biol Chem 1969;244:6388-6394.

2. Yoshida T, Kikuchi G. Purification and properties of heme oxygenase from pig spleen microsomes. J Biol Chem 1978;253:4224-4229.

3. Maines MD, Trakshel GM, Kutty RK. Characterization of two constitutive forms of rat liver microsomal heme oxygenase. Only one molecular species of the enzyme is inducible. J Biol Chem 1986;261:411-419.

4. Wu ML, Ho YC, Yet SF. A central role of heme oxygenase-1 in cardiovascular protection. Antioxid Redox Sign 2011;15:1835-1846.

5. Wang G, Hamid T, Keith RJ, et al. Cardioprotective and antiapoptotic effects of heme oxygenase- 1 in the failing heart. Circulation 2010;121:1912-1925.

6. Otterbein LE, Soares MP, Yamashita K, et al. Heme oxygenase-1: unleashing the protective properties of heme. Trends Immunol 2003;24:449-455.

7. Nath KA. Heme oxygenase-1: a provenance for cytoprotective pathways in the kidney and other tissues. Kidney Int 2006;70:432-443.

8. Kapturczak MH, Wasserfall C, Brusko T, et al. Heme oxygenase-1 modulates early inflammatory responses: evidence from the heme oxygenase-1-deficient mouse. Am J Pathol 2004;165:1045-1053.

9. Balla G, Jacob HS, Balla J, et al. Ferritin: a cytoprotective antioxidant strategem of endothelium. J Biol Chem 1992;267:18148-18153.

10. Sauer B, Henderson N. Site-specific DNA recombination in mammalian cells by the Cre recombinase of bacteriophage P1. Proc Natl Acad Sci USA 1988;85:5166-5170.

11. Loonstra A, Vooijs $M$, Beverloo HB, et al. Growth inhibition and DNA damage induced by Cre recombinase in mammalian cells. Proc Natl Acad Sci USA 2001;98:9209-9214.

12. Silver DP, Livingston DM. Self-excising retroviral vectors encoding the Cre recombinase overcome Cre-mediated cellular toxicity. Mol Cell 2001;8:233-243.

13. Naiche LA, Papaioannou VE. Cre activity causes widespread apoptosis and lethal anemia during embryonic development. Genesis 2007;45:768-775.

14. Buerger A, Rozhitskaya O, Sherwood MC, et al. Dilated cardiomyopathy resulting from high-level myocardial expression of Cre-recombinase. J Card Fail 2006;12:392-398.

15. Sohal DS, Nghiem M, Crackower MA, et al. Temporally regulated and tissue-specific gene manipulations in the adult and embryonic heart using a tamoxifen-inducible Cre protein. Circ Res 2001;89:20-25.

16. Feil R, Wagner J, Metzger D, et al. Regulation of Cre recombinase activity by mutated estrogen receptor ligand-binding domains. Biochem Biophys Res Commun 1997;237:752-757.

17. Metzger D, Clifford J, Chiba $H$, et al. Conditional site-specific recombination in mammalian cells using a ligand-dependent chimeric Cre recombinase. Proc Natl Acad Sci USA 1995;92:6991-6995.

18. O'Neal KR, Agah R. Conditional targeting: inducible deletion by Cre recombinase. Methods Mol Biol 2007;366:309-320.

19. Hall ME, Smith G, Hall JE, et al. Systolic dysfunction in cardiac-specific ligand-inducible MerCreMer transgenic mice. Am J Physiol Heart Circ Physiol 2011;301:H253-H260. 
20. Koitabashi N, Bedja D, Zaiman AL, et al. Avoidance of transient cardiomyopathy in cardiomyocyte-targeted tamoxifen-induced MerCreMer gene deletion models. Circ Res 2009;105:12-15.

21. $\mathrm{Xu} \mathrm{ZL}$, Mizuguchi $\mathrm{H}$, Ishii-Watabe $\mathrm{A}$, et al. Optimization of transcriptional regulatory elements for constructing plasmid vectors. Gene 2001;272:149-156.

22. Bolisetty S, Traylor AM, Kim J, et al. Heme oxygenase-1 inhibits renal tubular macroautophagy in acute kidney injury. J Am Soc Nephrol 2010;21:1702-1712.

23. Kim J, Zarjou A, Traylor AM, et al. In vivo regulation of the heme oxygenase-1 gene in humanized transgenic mice. Kidney Int 2012;82:278-291.

24. Clausell N, Butany J, Molossi S, et al. Abnormalities in intramyocardial arteries detected in cardiac transplant biopsy specimens and lack of correlation with abnormal intracoronary ultrasound or endothelia dysfunction in large epicardial coronary arteries. J Am Coll Cardio 1995;26:110-119.

25. Chen L, Knowlton AA. Mitochondria and heart failure: new insights into an energetic problem. Minerva Cardioangiol 2010;58:213-229.

26. Luptak I, Yan J, Cui L, et al. Long-term effects of increased glucose entry on mouse hearts during normal aging and ischemic stress. Circulation 2007;116:901-909.

27. Jameel MN, Zhang J. Myocardial energetics in left ventricular hypertrophy. Curr Cardiol Rev 2009;5:243-250.

28. Suliman HB, Carraway MS, Ali AS, et al. The CO/HO system reverses inhibition of mitochondrial biogenesis and prevents murine doxorubicin cardiomyopathy. J Clin Invest 2007;117:3730-3741.

29. Piantadosi CA, Suliman HB. Redox regulation of mitochondrial biogenesis. Free Radic Biol Med 2012;53:2043-2053.

30. Piantadosi CA, Carraway MS, Babiker A, et al. Heme oxygenase-1 regulates cardiac mitochondrial biogenesis via Nrf2-mediated transcriptional control of nuclear respiratory factor-1. Circ Res 2008;103:1232-1240.

31. Araujo JA, Meng L, Tward AD, et al. Systemic rather than local heme oxygenase-1 overexpression improves cardiac allograft outcomes in a new transgenic mouse. J Immunol 2003;171:1572-1580.

32. Sato K, Balla J, Otterbein L, et al. Carbon monoxide generated by heme oxygenase-1 suppresses the rejection of mouse-to-rat cardiac transplants. J Immunol 2001;166:4185-4194.

33. Hougen K, Aronsen JM, Stokke MK, et al. Cre-loxP DNA recombination is possible with only minimal unspecific transcriptional changes and without cardiomyopathy in Tg(alphaMHC-MerCreMer) mice. Am J Physiol Heart Circ Physiol 2010;299:H1671-H1678.

34. Ding HS, Yang J, Gong $\mathrm{FL}$, et al. High mobility box 1 mediates neutrophil recruitment in myocardial ischemia-reperfusion injury through toll like receptor 4-related pathway. Gene 2012;509: 149-153.

35. Oerlemans MI, Liu J, Arslan F, et al. Inhibition of RIP1-dependent necrosis prevents adverse cardiac remodeling after myocardial ischemia-reperfusion in vivo. Basic Res Cardiol 2012;107:270.

36. Kono H, Rock KL. How dying cells alert the immune system to danger. Nat Rev Immunol 2008;8:279-289.

37. Shi Y, Zheng W, Rock KL. Cell injury releases endogenous adjuvants that stimulate cytotoxic T cell responses. Proc Natl Acad Sci USA 2000;97:14590-14595.

38. Ishii KJ, Suzuki K, Coban C, et al. Genomic DNA released by dying cells induces the maturation of APCs. J Immunol 2001;167:2602-2607.

39. Hefeneider SH, Cornell KA, Brown LE, et al. Nucleosomes and DNA bind to specific cell-surface molecules on murine cells and induce cytokine production. Clin Immunol Immunopathol 1992;63: 245-251.

40. Fujioka D, Saito $Y$, Kobayashi $T$, et al. Reduction in myocardial ischemia/reperfusion injury in group $X$ secretory phospholipase A2-deficient mice. Circulation 2008;117:2977-2985.

41. Hiroi T, Wajima T, Negoro T, et al. Neutrophil TRPM2 channels are implicated in the exacerbation of myocardial ischaemia/reperfusion injury. Cardiovasc Res 2012;97:271-281.

42. Belcher JD, Mahaseth $\mathrm{H}$, Welch TE, et al. Heme oxygenase-1 is a modulator of inflammation and vaso-occlusion in transgenic sickle mice. J Clin Invest 2006;116:808-816.

43. Sass G, Soares MC, Yamashita K, et al. Heme oxygenase- 1 and its reaction product, carbon monoxide, prevent inflammation-related apoptotic liver damage in mice. Hepatology 2003;38:909-918.

44. Yet SF, Tian R, Layne MD, et al. Cardiac-specific expression of heme oxygenase-1 protects against ischemia and reperfusion injury in transgenic mice. Circ Res 2001;89:168-173.

45. Romson JL, Hook BG, Kunkel SL, et al. Reduction of the extent of ischemic myocardial injury by neutrophil depletion in the dog Circulation 1983:67:1016-1023.

46. Smith 3rd EF, Egan JW, Bugelski PJ, et al. Temporal relation between neutrophil accumulation and myocardial reperfusion injury. Am J Physiol 1988;255(5 Pt 2):H1060-H1068 